\title{
Desarrollo de competencias en el ámbito educativo: Definiciones conceptuales y operacionales
}

\section{Development of competences in the educationalfield: Conceptual and operational definitions}

\author{
Lucía Anderson $\square$ (iD Diana Londoño (iD) Gricel Martínez
}

Universidad Metropolitana de Educación, Ciencia y Tecnología. Ciudad de Panamá, Panamá

Recibido: 05/08/2021

Revisado: 20/09/2021

Aceptado: 29/11/2021

Publicado: 15/01/2022

\section{RESUMEN}

La educación de calidad se apoya hoy más que nunca en la formación por competencias. El avance y las exigencias de la sociedad actual, hacen necesario formar profesionales capaces de responder a las necesidades y retos del siglo XXI, sus expectativas laborales, sociales y personales. El artículo es el resultado de un análisis documental de distintos autores, expertos en el campo de la enseñanza por competencias. La aplicación de éstas por parte del docente en la práctica pedagógica, hace que se contribuya a la formación de individuos altamente calificados, integrales, con destrezas investigativas; cuyo aporte se ve finalmente reflejado en una sociedad competitiva, de vanguardia y equitativa. En conclusión, el artículo sostiene el postulado de que los conocimientos adquiridos previamente por el educando; son la fundamentación para que la enseñanza por competencias sea significativa, pertinente y esté presente a lo largo de su vida. En este sentido la educación por competencias va más allá del seguimiento temático y sustenta la flexibilidad curricular adentrando al estudiante en el nuevo ámbito contemporáneo e informacional. Palabras clave: Competencias, sistemas educativos, aprendizaje, estrategias, conocimiento previo.

\begin{abstract}
Currently, education relies more than ever on the developing of competence-based learning. The progress and demands of today's society poses the need of training professionals that are capable of responding to the 21st century challenges, jobs, social and personal expectations. This article is the result of a documentary analysis of different authors, and experts in the field of teaching by competences. This framework results in the formation of highly qualified, comprehensive, and research oriented individuals. Contributions are finally reflected in a competitive, vanguard, and equitable society. In conclusion, the article supports the thesis that learners' background knowledge is at the core of competency meaningful teaching, leading to relevant, lifelong learning skills. In this sense, the education by competencies model, goes beyond academic units and contents to enhance curricular flexibility, while introducing the student into the modern informational era.
\end{abstract} Keywords: Competences, educational systems, learning, strategies, knowledge previous. 


\section{INTRODUCCIÓN}

La globalización y sus exigencias conducen a una nueva forma de enseñar, que prepara al estudiante para realizar un trabajo de calidad, por medio de la adquisición de conocimientos nuevos, utilizando los previos y combinando sus actitudes y habilidades. Esta forma de enseñar busca brindarle al individuo un aprendizaje por y para la vida, a través de lo que se conoce como enseñanza por competencias.

La formación por competencias busca desarrollar un ambiente crítico en el ámbito escolar con la finalidad de impactar positivamente, el desempeño del estudiante a través de la puesta en práctica del conocimiento adquirido dentro y fuera del aula para que éste pueda autocorregirse y perfeccionar sus habilidades continuamente.

El aprendizaje basado en competencias rompe con los paradigmas tradicionales de una educación que se enfoca principalmente en el docente y en la repetición de contenidos extrapolados; transformándola en una formación por competencias, cuyo eje gira alrededor del educando. A nivel universitario la meta es el fortalecimiento del proceso investigativo, apoyado de contenidos procedimentales, conceptuales, y actitudinales pasando de impartir únicamente conceptos repetitivos, a un despertar de la motivación y la curiosidad intelectual del estudiante.

La formación docente desde el aprendizaje por competencias debe sufrir una transformación que equipe a los docentes con el conocimiento, las estrategias de enseñanza necesarias y recursos de aula para que éste incorpore cambios que involucren la asunción de nuevos hábitos en su forma de pensar y actuar. "El reconocimiento del impacto de sus acciones y de la relevancia de su función en la constitución de otro ser: el y la estudiante". Arreola Rico (2019). Así se logrará llegar a una sensibilización introspectiva y medir, de esta manera, el impacto que genera desde su práctica diaria.

\section{DESARROLLO}

Los sistemas educativos a nivel mundial están enfocados en proveer educación de calidad para todos los estudiantes con el fin de prepararlos para un futuro exitoso y competitivo. Se ha puesto la mira en el aprendizaje por competencias ya que este provee una educación integral para todos. Sarramona, Domínguez, Noguera y Vázquez, (2005 citado en Sánchez y Leicea, 2007) hablan del ímpetu con el que se está llevando a las aulas este aprendizaje. La idea es que la enseñanza por competencias este ligada a la preparación que se le debe dar al estudiante para enfrentarse al ambiente laboral. El aprendizaje por competencias los prepara a través de prácticas y actividades que se asemejan a la realidad para ponerlos en contexto y mirar su conocimiento y reacciones frente a la especificidad de cada situación.

Sánchez y Leicea (2007) denominan el aprendizaje basado en competencias como ABC y lo explican como el desarrollo de dos tipos de competencias, las genéricas y las específicas. Hablan de las transversales como instrumentos sistemáticos y describen las específicas como ajustadas a cada carrera. Ha sido la sociedad del conocimiento con su abrumador intercambio de información la que ha puesto a la educación cara a cara con retos que desembocan en la creación de estrategias para darle a los estudiantes la oportunidad de educarse en el nuevo contexto. Villa y Poble- 
te, (2007 citado en Sánchez y Leicea, 2007) enuncian que el progreso y la aplicación de las competencias abarcan mucho más que el saber, a la vez que integran de manera dinámica el conocer con el hacer para lograr que la formación tenga mayor relevancia y perdurabilidad.

Con el propósito de clarificar y dar contexto al proceso de enseñanza por competencias, debemos aclarar que existe una amplia gama de definiciones del término. Sánchez y Leicea (2007) las definen como la mezcla de actitudes, valores, maestría y estrategias utilizadas durante la actuación académica y profesional. Una competencia es el nivel de rendimiento de una persona en un ambiente diverso y real que permite la puesta en práctica del conocimiento a través de destrezas aplicadas. Por otro lado, Rodríguez y Feliu (1996 citados en Valiente y Galdeano 1996) dicen que las competencias son agrupaciones de saberes, destrezas, aptitudes y comportamientos que tiene una persona y que le permiten desempeñar una actividad de manera exitosa. Para Ansorena Cao (1996 citado en Valiente y Galdeano, 1996) es una cualidad del comportamiento de la persona, puede ser considerada una característica de su conducta que va dirigida a la ejecución de la tarea y se puede catalogar de manera confiable y certera.

A nivel universitario se puede decir que las competencias son un grupo de cualidades relacionadas con los saberes que posee el estudiante, su puesta en práctica, su actitud y su responsabilidad, basados en un programa académico que la universidad le ofrece. Asimismo, las competencias a nivel universitario se refieren a la forma en que el individuo es capaz de desarrollarse durante todo el proceso educativo, utilizando sus conocimientos para lograr el éxito académico. Para Cano (2008, citado en Martínez, Cegarra y Rubio, 2012) las competencias poseen tres características, a saber:

a) Están formadas por conocimientos conceptuales, procesos y actitudes, mismos que a la vez trascienden. Ser competente significa ser capaz de seleccionar entre todo el conocimiento que el individuo posee, el necesario para resolver un problema, una situación, o un reto del momento.

b) Las capacidades están relacionadas con la personalidad del individuo y pueden ser aprendidas, evolucionar por medio de la formación inicial, inculcar la educación permanente $\mathrm{y}$ experimentar durante toda la vida. En otras palabras, el individuo nunca es competente pues está en constante aprendizaje y evolución.

c) A través de su puesta en práctica las competencias cobran sentido cuando el individuo reflexiona sobre el proceso llevado a cabo durante la resolución de una situación o de un problema determinado. Gracias a esta reflexión, las competencias no se vuelven estandarizadas pues se logran reorientar las acciones utilizadas, a fin de que el paso siguiente sea mejorado.

Schunk (2008 citado en Martínez, Navarro, y Sánchez, 2012) expone que la adaptación de la enseñanza por competencias implica que el profesor se enfoque en el aprendizaje o sea el proceso de recibir, analizar, generar relaciones y guardar información que se presenta durante la acción cognitiva. Es importante que los estudiantes aprendan a ser autónomos en el trabajo y se vinculen cooperativamente en proyectos que reflejen su realidad. 
González y Ramírez, (2011 citado en Forero, 2020). Dice que a nivel profesional la enseñanza por competencias impulsa el desarrollo y genera procesos de calidad de manera que el educando pueda dar soluciones a los requerimientos sociales a través del conocimiento que conlleva su profesión.

Enfocarse en la educación por competencias permite que los estudiantes fortalezcan sus capacidades durante el proceso de aprendizaje, a la vez que pueden adquieren flexibilidad frente a los diferentes retos que les presenta la sociedad. Spencer y Spencer (1993) definen competencia como un rasgo tácito en un ser humano, relacionado con su actuación frente a una asignación. $\mathrm{O}$ sea, un conjunto de conductas que se amoldan a una circunstancia.

Los rasgos tácitos pertenecientes a las competencias están estrechamente ligados a la personalidad del educando y le ayudan a decidir su reacción frente a cualquier situación, en este caso académica o profesional. Estos rasgos son: motivos, lo que el educando piensa con frecuencia. Son las metas que desea alcanzar (notas altas, reconocimiento, y otros) estos pueden ser explícitos o implícitos. Rasgos de la personalidad, las peculiaridades físicas de reacciones constantes frente a una situación o al recibir información. En este rasgo se ve cómo los educandos reaccionan de diferente manera y tiempo a estímulos relacionados con su quehacer estudiantil y se puede visualizar la iniciativa de cada uno, al recibir los requerimientos del educador o de los otros estudiantes. El auto concepto, denota la postura, valores, o la imagen que cada estudiante tiene de sí mismo. Se definen aquellos que son líderes, los que no desean llamar la atención o aquellos que valoran la calificación como lo más importante, frente a los que aprecian las relaciones sociales, sobre todo. El conocimiento, o datos que posee el estudiante acerca de conceptos que están contenidos dentro del plan de estudios, su habilidad, destreza o capacidad al desempeñarse en una actividad relacionada con su campo de experiencia, sea ésta física o no. Las competencias son de gran ayuda ya que facilitan la identificación de destrezas o deficiencias al desarrollar una actividad. Estas destrezas se miden con herramientas basadas en estándares específicos.

Es importante entender que la actualización en el uso de competencias es primordial. Según Díaz (2005) las competencias que posee un educando "no son para siempre" lo que era apto para el pasado, ya no es adecuado hoy en día. En este sentido, en los últimos años la educación por competencias se convierte en una estrategia de gran importancia, al fusionarse con las TIC, como apoyo a nivel educativo con el fin de continuar el proceso de enseñanza - aprendizaje. De este modo, se desprende el argumento que la educación por competencias debe mantenerse en constante actualización y favorecerse de los cambios que trae consigo el desarrollo de la información y el avance tecnológico.

Ahora bien, la manera como el educando desarrolle su competencia está relacionada con el medio ambiente en el que se desenvuelve, el manejo que tenga del tema y sus propios rasgos de personalidad. Se ven implicadas también con sus intenciones, limitaciones, motivaciones y rendimiento. De lo anteriormente expuesto, se puede desprender que las competencias son el conjunto de conocimientos, actitudes y habilidades que tiene un individuo para realizar una tarea de manera exitosa, efectiva y responsable, teniendo la capacidad de ponerlas en práctica en distintos contextos. 
También se puede decir que las competencias son observables por medio del desempeño de trabajos y aunque no están ligadas al éxito, éstas ayudan a obtenerlo. Gracias a una reflexión profunda sobre el proceso desarrollado durante la solución del problema, éste puede mejorarse hasta alcanzar su perfeccionamiento.

Las instituciones educativas a nivel superior, se han visto en la necesidad de ofrecer a los estudiantes programas académicos centrados en las demandas laborales y por tanto, en las competencias. El término competencias tiene sus orígenes en el ámbito educativo desde hace aproximadamente cinco décadas. Las mismas, son el resultado de distintos factores que convergen: la expansión que ha sufrido el proceso de globalización y el nacimiento de lo que se conoce como la sociedad del conocimiento. Por una parte, este concepto fue renombrado por las teorías de la comunicación de Noam Chomsky en el año 1965. Según el autor, la palabra competencias está vinculada a la forma en que las personas se adueñan del lenguaje y la forma en que lo utilizan para llevar a cabo el proceso comunicativo. Por otra parte, Barbero (2003 citado en Valiente, 2009) menciona que la reingeniería considera el término como la suma de las habilidades del saber-hacer con las de saber competir y obtener mayores resultados económicos en un ambiente competitivo. En resumen, la lingüística relaciona el concepto competencias con las habilidades intelectuales mientras que la reingeniería lo utiliza para referirse a la habilidad del individuo para producir riqueza y ser competitivo.

A partir de los años treinta, el término competencias se introdujo en el ámbito educativo en los Estados Unidos cuando, según Posada (2004, citado en Valiente, 2009), se propo- ne una educación basada en las competencias. Alrededor de los años sesenta, se hizo referencia al término competencias laborales en el entrenamiento de los trabajadores utilizando técnicas conductistas. A nivel empresarial la formación por competencias tiene un enfoque funcional ya que considera los conocimientos, las actitudes y las aptitudes como las bases de éstas, las cuales deben ser estimuladas para obtener mayor efectividad productiva. Hoy en día, las teorías modernas se basan en el enfoque dinámico, el cual relaciona el término competencia con el nivel de funcionalidad de la persona en su actuar (su responsabilidad, su interactuar con perseverancia y con autonomía y su flexibilidad) a la hora de realizar un trabajo, más que a un simple listado de sus atributos y de sus cualidades que la categorizan como calificada para desempeñarse eficientemente.

Las múltiples dimensiones de las competencias y la proyección del individuo en las distintas profesiones hacen que éstas se vuelvan intrincadas. No obstante, en un escenario complejo como éste, las competencias hacen frente a la situación mediante la movilización de distintos recursos. Por tanto, la formación por competencias busca que las personas puedan desarrollar una gran variedad de capacidades, que les ayuden a aprender y a desaprender durante toda su vida para que tengan la capacidad de adaptarse a las distintas situaciones cambiantes que se le puedan presentar. Cabe señalar que a nivel superior se debe promover el desarrollo de competencias profesionales por medio del saber, saber hacer y saber ser y estar; esto, según Echeverria (2002, citado en Martínez, 2012) debe "asegurar o acreditar el saber profesional".

Ahora bien, para lograr probar la adquisición de distintas competencias por parte 
del estudiantado, se hace necesaria la implementación de un sistema de evaluación basado en los resultados de este aprendizaje. Este sistema permitirá que se logre monitorear el proceso desarrollado como eje central para tomar decisiones y lograr un mejoramiento. Según lo plantea Moreno (2012), La evaluación educativa ha sido conocida tradicionalmente como el medio por el cual el docente registra el avance cognoscitivo en cuanto a determinados productos de aprendizaje, suele ser unilateral y enfocado en medir el alcance de logro del estudiante. En el método tradicional, el centro del proceso es el maestro y los instrumentos que permiten revisar el impacto o no del proceso de formación, mismo que gira alrededor de pruebas estandarizadas. Sin embargo, en el proceso de enseñanza-aprendizaje a través de competencias, la evaluación gira alrededor del rol activo del estudiante. Éste, indiscutiblemente, es el autor y partícipe de su evaluación, pues es quien tendrá la tarea de demostrar las capacidades adquiridas sin que haya otra persona que pueda hacerlo por él.

Como lo señala Moreno (2012), al facilitar diversos ejercicios de evaluación en contexto, se le otorga al discente los medios para demostrar el grado de dominio de las competencias antes trabajadas en el ámbito escolar. En este sentido, la evaluación formativa a través de proyectos, ejercicios grupales, actividades escritas y orales, portafolios, exámenes, entre otros, aportarán múltiples escenarios para que los estudiantes puedan probar el nivel de competencia alcanzado. Así como la evaluación es multiforme, también deberá serlo la implementación de diversos acercamientos desde la co-evaluación, autoevaluación y heteroevaluación (Moreno, 2012).
Algunos métodos tradicionales, que no necesariamente son de uso exclusivo de la formación educativa por competencias, aún se encuentran vigentes en su aplicabilidad para el desarrollo de habilidades que se transfieren del ámbito escolar a la vida cotidiana. Para comenzar, se habla de la observación como una técnica que permite verificar la propiedad con la que se desempeña la persona al poner en práctica ciertas habilidades en su contexto. La observación ha de ser objetiva, sistemática y precisa de tal manera que proporcione información del nivel de dominio de las competencias en el individuo.

Por otra parte, la técnica de la entrevista formal o informal, es un ejercicio planeado y dotado de intencionalidad que supone una conversación entre las partes, en igualdad de condiciones. Ésta, ha de propiciar espacios de escucha, confianza y naturalidad que le permiten al estudiante y al maestro generar un espacio armonioso de respeto, en el cual se habla de los logros alcanzados, a la vez que se plantean posibles dificultades en el proceso. Según Poblete Ruiz et al. (2015), en el ámbito educativo, la entrevista vinculada a la evaluación permite ver esta etapa como una parte del proceso formativo más no como su único fin. Es decir, la evaluación por medio de la entrevista es un escalón más en el proceso formativo.

Adicionalmente, un recurso más de evaluación son las actividades de aula basadas en proyectos. Estas poseen la virtud de ser aplicables dentro y fuera del aula de clase. Diaz Barriga (2006) establece la multiplicidad de formas que puede tomar un proyecto y que bajo condiciones de complejidad permite el alcance de los logros previamente establecidos. 
Los proyectos trascienden las paredes del aula de clase y permiten la aplicabilidad de las competencias en la vida real. Su autenticidad genera un interés único en el educando puesto que ve la función de los proyectos de aula en pro de sí mismo y de la sociedad.

Otro recurso de evaluación por competencias es el aprendizaje basado en problemas, (ABP) definido por McGrath (2002), como el método de aprendizaje colectivo que emplea situaciones problémicas del diario vivir, para promover habilidades de resolución de conflictos y así desarrollar conocimiento en áreas particulares. Éste se orienta en el pensamiento constructivista dado que parte de una situación problémica y de forma colaborativa se buscan soluciones y/o la comprensión del mismo. Aquí entra en juego la interdisciplinariedad que debe llegar a la vinculación de habilidades generales y específicas en los educandos. El ABP pretende descentralizar la enseñanza tradicional a partir de lecturas y lecciones, llevándola a un trabajo colaborativo por medio de objetivos concertados, diálogo entre pares, formulación de hipótesis y un plan de trabajo que hace de cada integrante del equipo un elemento único e indispensable. Según lo planteado por Moreno (2012), son tres los elementos claves para la evaluación por medio de ABP: la reflexión cooperativa sobre el problema, el estudio auto dirigido de la temática que permita entender y solucionar el planteamiento y la aplicación en grupo de los conocimientos adquiridos.

Otro método para evaluar por competencias es el estudio de casos en el que los estudiantes se familiarizan con situaciones que se asemejan a problemáticas de su entorno. Éstas cumplen la función de escenarios reales o hipotéticos, donde los discentes ana- lizan normas de cada contexto y, al mismo tiempo, fortalecen sus habilidades sociales.

Las simulaciones son otra herramienta que utiliza la tecnología para crear diferentes panoramas y recursos novedosos que le permitan al estudiante más práctica y exponerse a las competencias en cuestión. Es indispensable que las simulaciones tengan un alto contenido de aproximación a la realidad del discente, de tal manera que sean aplicables a su día a día. De esta forma se pretende modelar y reforzar las cualidades de un ciudadano y profesional íntegro. Para utilizar las simulaciones como herramientas de evaluación es indispensable que éstas culminen con una evaluación y autoevaluación de lo aprendido, mismas que otorgan el componente reflexivo de la formación a través de competencias.

Por lo que se refiere al aprendizaje cooperativo, Sharan (1980) plantea éste como una serie de estrategias sistemáticas que se pueden emplear en cualquier área del conocimiento y nivel de escolaridad. Dos características particulares del aprendizaje cooperativo son, por un lado, la creación de grupos heterogéneos (según los niveles de desempeño, sexo, raza, edad, entre otros); por el otro, generar en los equipos de trabajo un ambiente positivo de recompensa que les invite a alcanzar los objetivos propuestos. Se destaca como un valor agregado del aprendizaje cooperativo el hecho de que permite, no sólo demostrar el nivel de desempeño según las competencias planteadas, sino que también se desarrolle un ambiente idóneo para fortalecer las competencias socio afectivas entre pares. Otras ventajas agregadas del aprendizaje cooperativo radican en el desarrollo del conocimiento, la negociación de reglas y normas, el fortalecimiento de valores como la responsabilidad, respeto mutuo y tra- 
bajo consensuado hacia un objetivo en común.

El portafolio es otra forma de evaluación por competencias que consiste en una serie de documentos que muestran el avance de los estudiantes. Algunos ejemplos de trabajos del discente, dentro o fuera del contexto de aula, pueden ser cartas, ensayos, fotografías, dibujos, presentaciones orales, entre otros. Una de las ventajas del portafolio es que el estudiante puede elegir qué documentos va a incluir, puede agregar comentarios, discusiones con el profesor e incluso influir la forma en que su portafolio será evaluado. Otra ventaja, en el campo de las ciencias, es que permite el pensamiento científico, pues proporciona las evidencias necesarias para probar o debatir algo. Esta herramienta de evaluación explica, provee alternativas, ofrece nuevas opciones de actuación y aclara conceptos a través de los trabajos que el estudiante selecciona. Según Barbera (2005), en el campo de las ciencias sociales se incrementa el entendimiento y el análisis a profundidad de nuevos conceptos y hechos, su explicación, así como los argumentos de perspectivas personales que han sido adoptadas. La construcción del portafolio sigue un esquema básico. Primero, debe comprenderse el fenómeno (esto se logra mediante la observación, la decodificación, el análisis y la síntesis). Luego, se debe realizar una selección significativa de habilidades (de discriminación y de valoración) y una defensa explicativa (demostrada por medio de la composición y la argumentación). Durante la reflexión, el estudiante debe tomar conciencia de su propio proceso de aprendizaje, a través de las habilidades metacognitivas.

De lo anterior se desprende que el uso del portafolio le ofrece al estudiantado dos ventajas. La primera, es un alto grado de motivación pues el estudiante ve plasmada la continuidad de su trabajo, su conocimiento y creatividad. La segunda ventaja es que los criterios de evaluación los conoce desde el día uno al igual que su transparencia para que según Barbera (2005), el discente pueda comprenderlos. Dentro de estos criterios están la importancia, la coherencia, la intención, la auto-evaluación, entre otros. Se recomienda que el docente ofrezca distintas formas de evaluación dentro del mismo portafolio.

Las rúbricas son una herramienta formada por pautas que especifican los parámetros bajo los cuales se evalúa un diseño o proyecto; permitiendo al estudiante entender lo que se espera de su trabajo a través de una retroalimentación clara. Se manejan dos clases de rúbricas: las analíticas y las holísticas, que a su vez se pueden constituir en genéricas (enfocadas en las competencias transversales como la oralidad y la escritura) o específicas a determinada materia u ocupación. La función primordial de una rúbrica es la evaluación veraz y opcional del estudiante, de su trabajo y rendimiento de forma imparcial y estable, además de generar una retroalimentación que tenga significado para el dicente en un tiempo corto. Según Goñi (2005), las rúbricas son instrumentos que apoyan el pre, el post y la evaluación continua de una unidad; ya que le permiten al educador redireccionar las metas de aprendizaje y la manera en que se evaluará. Al mismo tiempo generan un espacio de comunicación entre docente y estudiante con el fin de proveer contexto sobre el trabajo realizado de manera constante, generando en el estudiante la capacidad de autoevaluarse. Se aconseja la aplicación de las rúbricas con el fin de emitir observaciones acerca de un proyecto o trabajo del estudiante a nivel oral o escrito. También se pueden emplear en trabajos técni- 
cos o científicos. Lo más importante para el uso de una rúbrica es enfocarse en el objetivo a evaluar y en el tema de aprendizaje planteado.

Aunque se conocen las pruebas escritas como elementos típicos de la enseñanza tradicional, éstas no se descartan de su aplicabilidad en el aprendizaje a través de competencias. No existe un veto en el uso de las pruebas escritas cuando se busca formar educandos competentes; más bien, se deberá analizar con detenimiento el tipo de preguntas que se formulen y el propósito por el cual se recurre a la aplicación del examen. Si el maestro usa la prueba escrita como mecanismo para medir su instrucción y redireccionar a los educandos, estas pruebas serán de real beneficio para la formación de competencias. Por el contrario, es necesario evitar la aplicación de exámenes que busquen la recopilación de respuestas automáticas y memorísticas. Se destaca que la aplicación de pruebas escritas, por lo general, apunta a los niveles más básicos en la taxonomía del aprendizaje.

\section{CONCLUSIONES}

El aprendizaje basado en competencias busca el desarrollo y la implementación de los conocimientos previos y nuevos, las actitudes y habilidades del individuo para resolver situaciones problemáticas y retos, permitiendo al estudiante tomar decisiones, con base en el dominio de su propio repertorio.
La enseñanza por competencias proporciona las herramientas necesarias para que la persona genere un aprendizaje imperecedero. Además, la auto-reflexión le permite optimizar sus habilidades durante el proceso, así como identificar las debilidades con respecto a éste para perfeccionarlo.

El uso de las competencias en educación, le posibilita al docente enfocarse primordialmente en el proceso de aprendizaje, facilitando un enfoque claro en la planeación de éstas con el fin de apoyar las necesidades individuales de los estudiantes. Este planeamiento curricular debe ir acompañado de un proceso evaluativo y flexible por parte del docente, ya que exige la modificación del currículo para evitar la saturación temática.

El docente debe seleccionar contenidos esenciales que permitan formar ciudadanos activos, capaces de desempeñarse con éxito en su profesión. Es importante generar un espacio de tiempo que permita el desarrollo de las competencias del estudiantado tanto dentro como fuera de la institución educativa.

Los retos modernos han colocado al alumnado en una posición de competitividad y desafíos, por lo cual el educador debe estar capacitado para cubrir las nuevas necesidades del estudiante y así éste pueda desempeñarse y satisfacer las exigencias del mercado laboral moderno de manera exitosa.

\section{REFERENCIAS BIBLIOGRÁFICAS}

Arreola Rico, R. L. (2019). Formación y evaluación docente basada en un perfil por competencias. Una propuesta desde la práctica reflexiva. Revista Educación, 43(2), 30. https://doi.org/10.15517/revedu.v43i2.30898 
Castaño, G. y Macías, V. (2005). Una mirada a las competencias como referente de formación integral para administradores de empresas de la Universidad Nacional Sede Manizales. Revista EAN, 5-25. DOI https://rb.gy/ef7gun

Castelló, M., Monereo, C. y Gómez, I. (2009). Las competencias de los alumnos y su evaluación. En Monereo, C. (Coord.). Pisa como excusa. Repensar la evaluación para cambiar la enseñanza (pp. 33-53). Graó. https://rb.gy/cenp5q

De Miguel Díaz, M. (2005). Modalidades de enseñanza centradas en el desarrollo de competencias. Orientaciones para promover el cambio metodológico en el Espacio Europeo de Educación Superior. https://rb.gy/k4j8dm

Díaz Barriga, A. (2006). El enfoque de competencias en la educación. ¿Una alternativa o un disfraz de cambio? Perfiles Educativos, 28 (2), 7-36. https://rb.gy/ppis5x

Eisner, E. (1998). El ojo ilustrado. Indagación cualitativa y mejora de la práctica educativa.

Paidós. https://rb.gy/foanb8

Forero, J. C. G. (2020). Fortalecimiento de competencias mediante educación virtual en un contexto de pandemia: Resultados de una experiencia Naydú Acosta Ramírez.

REVOLUCIÓN EN LA FORMACIÓN Y LA CAPACITACIÓN PARA EL SIGLO XXI, 3(1), 263-269. https://rb.gy/z5b2kb

Martínez, A. M., Navarro, J. M. C., y Sánchez, J. A. R. (2012). Aprendizaje basado en competencias: Una propuesta para la autoevaluación del docente. Profesorado, Revista de currículum y formación del profesorado, 16(2), 325-338. https://rb.gy/p3tvp6

McGrath, D. (2002). Teaching on the Front Lines: Using the Internet and ProblemBased Learning to Enhance Classroom Teaching. Holist Nurse Pract, 16 (2), pp. 5-13 DOI: 10.1097/00004650-200201000-00004

Moreno, T. (2012). La evaluación de competencias en educación. Sinéctica, 39. http://www.sinectica.iteso.mx/index.php?cur=39\&art=39_09

Poblete-Ruiz, M., Fernández, D., Campo, L., y Nöel, M. (2015). La entrevista como herramienta para la evaluación de las competencias genéricas: formación y construcción de un consenso sobre evidencias. Revista Complutense de Educación, 27(2), 457-476. https://doi.org/10.5209/rev_rced.2016.v27.n2.46375 
Sánchez, A. V., y Leicea, O. V. (2007). El aprendizaje basado en competencias y el desarrollo de la dimensión social en las universidades. Educar, 40, 15-48. https://rb.gy/rfhljw

Sharan, S. (1980). Cooperative learning in small groups: Recent methods and effects on achievement, attitudes and ethnic relations. Review of Educational Research, 50, 241-272. https://rb.gy/rqvk19

Valiente Barderas, A., y Galdeano Bienzobas, C. (2009). La enseñanza por competencias. Educación Química, 20(3), 369-372. https://doi.org/10.1016/s0187-893x(18)30038-7 